\section{GW23-e1751 AWARENESS, TREATMENT, AND CONTROL OF HYPERTENSION IN CHINA: FINDINGS FROM 2010 CHINA CHRONIC DISEASE AND RISK FACTOR SURVEILLANCE}

doi:10.1136/heartjnl-2012-302920d.2 in women $(84.9 \%, 95 \%$ CI $83.4 \%$ to $86.3 \%)$ than in men $(75.8 \%$, $95 \%$ CI $73.9 \%$ to $77.7 \%, \mathrm{P}<0.01$ ), and increased with age ( $\mathrm{P}$ for trend $<0.01)$. Neither the proportions of treated individuals varied by rural/urban residency ( $80.8 \%$ vs $80.2 \%, p=0.72$ ), nor differed among geographical regions $(80.3 \%$ for east, $82.1 \%$ for middle, and $78.1 \%$ for west, $\mathrm{P}$ for difference $=0.11$ ). As opposed to treatment, the percentages of controlled BP decreased with age ( $\mathrm{P}$ for trend $<0.01)$. No significant difference of BP control existed between men $(19.1 \%, 95 \%$ CI $17.3 \%$ to $21.0 \%)$ and women $(17.9 \%, 95 \% \mathrm{CI}$ $16.2 \%$ to $19.6 \%, p=0.17)$. A strong rural-urban disparity of $\mathrm{BP}$ control was observed: $15.3 \%$ (95\% CI $13.6 \%$ to $17.0 \%$ ) in rural areas and $23.9 \%(95 \%$ CI $21.0 \%$ to $26.7 \%)$ in urban areas $(\mathrm{P}<0.01)$. Similar control of BP were found among geographical regions (19.5\% for east, $16.8 \%$ for middle, and $18.9 \%$ for west region, $\mathrm{p}=0.28)$.

Conclusions Awareness of hypertension in Chinese adults with hypertensive condition had increased greatly since 2002, but was far from enough. Adequate control of blood pressure remained at a quit low level in this decade despite treatment was as high as more than $80 \%$. Improving awareness in a wider population and enhancing efficacy of treatment should still be the very focus of national hypertension programs.
Yichong Li, Limin Wang. National Center for Chronic and Non-communicable Disease Control and Prevention, Chinese Center for Disease Control and Prevention

Objectives We aimed to examine the awareness, treatment, and control of hypertension in Chinese adults and to explore their difference among subpopulations.

Methods The 2010 China Chronic Disease and Risk Factor Surveillance was carried out from August to November 2010 nationwide in mainland China. A total of 98548 respondents sampled by multistage clustering sampling design were included in the analyses. All respondents had their blood pressure (BP) measured 3 times successively by using unified electronic BP monitor. The average of last 2 measures was used for analyses. Information on history and treatment of hypertension was collected by questionnaire-based interview. The awareness, treatment, and control of hypertension were examined by age, sex, rural/urban residency and geographical regions. For comparisons of percentages between subgroups, Rao-Scott $\chi^{2}$ test was used. Logistic regression was used to test trend over age. Taylor series linearisation was applied to estimate CIs. All calculations were carried out with complex weights in SAS V.9.3.

Results Overall, of individuals with hypertension, 35.8\% were aware of their condition, which was much higher than the estimate (24\%) from 2002 China National Nutrition and Health Survey (CNNHS). More women (39.1, 95\% CI 37.3\% to $40.9 \%$ ) were aware of their hypertensive status than were men $(32.8,95 \%$ CI $31.0 \%$ to $34.6 \%$; $\mathrm{P}<0.01)$. Awareness of hypertension increased with age ( $\mathrm{p}$ for trend $<0.01$ ). Urban residents with hypertension (41.1\%, $95 \%$ CI $38.4 \%$ to $43.7 \%$ ) had a higher percentage of being aware their condition than did rural residents $(33.2 \%, 95 \% \mathrm{CI}$ $31.1 \%$ to $35.4 \% ; \mathrm{P}<0.01)$. Awareness of hypertension decreased from $38.4 \%$ (95\% CI $35.7 \%$ to $41.1 \%$ ) in east, $35.3 \%$ in middle ( $95 \%$ CI $32.2 \%$ to $38.4 \%$ ), to $31.6 \%$ in west region of China $(95 \%$ CI $28.8 \%$ to $34.5 \%$, p for difference $<0.05)$.

Four in five $(80.4 \%, 95 \%$ CI $79.0 \%$ to $81.9 \%)$ received prescribed antihypertensive medications, but only $18.5 \%$ (95\% CI $17.0 \%$ to $20.0 \%$ ) had their BP controlled among individuals who were aware of their hypertension. The treatment and control of hypertension were quite similar to those reported by 2002 CNNHS (78\% and $19 \%$, respectively). Antihypertensive treatment was more common 